\title{
ESTIMATION OF CONSERVATISM OF CHARACTERS BY CONSTANCY WITHIN BIOLOGICAL POPULATIONS
}

\author{
JAMES S. FARRIS \\ Museum of Zoology, The University of Michigan, Ann Arbor
}

Accepted March 30, 1966

The concept of conservatism is basic to much of taxonomy. A conservative character is usually defined as one that has changed more slowly than a non-conservative one during phylogeny. The reliability of a character for placing organisms properly in evolutionary schemes is also included in conservatism. It is argued quite validly that a character which changes slowly is the best kind of character for discerning the evolutionary relationships of organisms. This is true in practice because of the very incompleteness of the historical record of phylogeny. When not all the forms of a line are known, there will be gaps in the record of rapidly-changing characters. But slowly-changing ones may still have a fairly complete series represented, even if only a few forms are known. The patterns of evolutionary relationship are easier to trace in characters that evolve slowly.

It is usually assumed that not all characters are equally reliable, and many taxonomic problems can be reduced to the process of recognizing the most reliable ones. It has long been the practice to select conservative characters by the criteria of constancy within known groups and estimated biological importance. This approach has been criticized by numerical taxonomists (Sokal and Sneath, 1963), who point out that biological importance is seldom known, and that calling a character conservative if it is constant within a known group constitutes circular reasoning, if the group was constructed on the assumption that the character was conservative.

It is not the concept of conservatism itself that is invalid, but only the means that have been suggested for finding con- servative characters. There is little doubt that if a character is constant throughout a group that is natural in an evolutionary sense, then that character is a more reliable indicator of evolutionary relationship than is a character that varies widely in the same group. I shall present an argument to show how it may be inferred which characters are most likely to be conservative.

Suppose that a given character is conservative in that it varies little in a given natural group. The variability of that character between the populations of that hypothetical group can only be small if the variability within single biological populations is also small. Otherwise random variation alone would be expected to produce wider variation within the group.

Now it has been argued that the fitness of a population is maximized when the population is carrying as much heterogeneity as it can (Mayr, 1963). But then we expect a character to vary little within populations only if the centripetal selective forces acting on it are relatively intense. Thus we would expect that a conservative character would be characterized by a low variability within populations, in turn due to heavy selection on that character.

On the other hand, it seems fair to say that a character with high centripetal selection acting on it is of great adaptive importance to the organism, and conversely. Then we expect a character with low variability within populations to be conservative, at least in the sense that it is of great biological importance: it is important to the survival of the organism. It is likely that it would be relatively difficult for such a character to change during the course of evolution. Because of the intense centripetal selection acting on it, a population un- 
dergoing modification in such a character would face a high risk of extinction. Changes in other characters with a higher variability would be much easier and safer, and could be expected to be much more common. Then we would expect that for each state of the conservative character there would be a group of species that had radiated adaptively in other characters while holding the conservative one constant. It seems proper to refer to such groups of species as natural groups in an evolutionary sense. This is not meant to imply a monothetic concept of "natural group," but merely that if a character is conservative, it is expected to index the membership of a group that is natural in a phylogenetic sense.

This argument shows that the principles of population genetics predict that the two criteria of conservatism, constancy over groups and biological importance, will be equivalent ways of selecting conservative characters. Of more interest from the standpoint of inference is the middle part of the syllogism. Even when we do not know the real natural groups or the true biological importance of the characters, we can select the most likely conservative characters by choosing those with the lowest variability within biological populations. Similar results have been suggested by previous workers. Mayr (1963) in his discussion of phenotypic canalization points out that the canalization phenomenon occurs when tremendous selective importance is attached to a particular state of some character. $\mathrm{He}$ postulates that the effects may extend far into the subsequent development of a population, holding the canalized character constant while other characters change. Mayr was of the opinion that the phenomenon of canalization at the population level might well be the explanation for the observed constancy of some characters over widely diversified evolutionary groups. Fisher (1930) pointed out that a character with high variability in the population must either be undergoing rapid evolution or be of little selective importance. This implies that a character with much variability in the population is not expected to be conservative. Guthrie (1965) showed in an analysis of fossil and recent Microtus that characters undergoing rapid evolution tend to increase in variability within the population. He also pointed out that characters more variable within populations in given taxonomic groups tend also to be more variable between populations in those groups.

It seems clear that the conservatism of a character can be reliably estimated by its constancy within discrete populations. Variability within particular populations can easily be measured by the sample variance. It would seem most reasonable to calculate constancy-and therefore estimated conservatism-by the reciprocal of the sample variance of a random sample drawn from the population. In taxonomic procedures, characters could then be weighted according to their estimated conservatism. If the taxonomic statistic consisted of a set of unit character differences between populations, each difference could simply be divided by the corresponding intrapopulation standard deviation (or each squared difference could be divided by the sample variance). If such standardized differences were then given equal weight, the whole analysis would in effect be weighted in favor of the conservative characters.

Groups constructed using characters weighted according to constancy within populations can be considered to conform to the evolutionary population theory of the New Systematics. But it is debatable whether the taxonomic scheme arrived at is actually dependent on the above argument. Many statistics now in use in taxonomy use a similar method of weighting anyway, for reasons rooted in probability theory. Given a procedure that does weight in this way, the argument does show that the results obtained can be expected to be biologically reasonable. This was indeed found by Olson (1964) in the case of the Generalized Distance, used on fossil oreodonts. In any event, the logic certainly is 
not circular, unless the resulting taxonomic scheme is itself used to support the idea that characters constant within populations tend also to be conservative in transpecific evolution.

Sokal and Sneath (1963) suggest weighting characters according to their constancy between the Operational Taxonomic Units (OTU's) used in a given study. It is important to note that this is not the same procedure as the one presented above. It does not seem likely that their method would give good results. For example, if an OTU differs strikingly from others in a series of characters, those divergent characters will be given low weight, since the variability between OTU's is high. This could lead to as serious an error as a gross underestimation of the magnitude and importance of the separation of the aberrant OTU. If there is much taxonomic diversity represented in the collection of OTU's studied, no particular relationship is expected between the conservatism of a character and its variability over the whole set of OTU's. This procedure, termed "standardization by rows," was proposed by Sokal and Sneath in order to simplify calculation of correlation coefficients and to insure that all characters would have an equal effect on the analysis. But one must conclude for the reasons I have given that this form of weighting does not seem very promising for evolutionary studies.

A character may be unreliable for taxonomic purposes, even though it is conservative according to the criterion of constancy within populations. Characters in which convergence occurs may be very conservative, yet have little power properly to place organisms in evolutionary groups. Such characters are not unreliable in generalbut only in some comparisons. For example, body form is a poor means by which to distinguish ichthyosaurs from fishes, but it is a perfectly reliable way to tell them from pterosaurs. Examples of characters showing convergence as well as low variability within populations should not, therefore, be considered to invalidate the idea that the characters with the lowest variability within populations are generally the most conservative ones. Convergence between distantly related organisms can occur in characters that are of the greatest usefulness in working out evolutionary patterns. The problem of avoiding error due to convergence is quite distinct from that of finding conservative characters, and will be treated separately in a future paper.

With the exception of characters that are considered poor because of convergence, estimating conservatism by constancy within populations correlates well with classical taxonomic practice. Characters diagnostic for large groups turn out also to have low variability within populations belonging to those groups. Except for certain convergent characters with low intrapopulational variability, almost all characters with low variability within populations are useful in working out evolutionary patterns.

In some taxonomic problems, notably those involving the evolutionary patterns within small groups, such as species-groups or genera, it is often difficult, using conventional methods, to make decisions as to which characters are the best. Presumably it is the best procedure to ignore "poor" characters, but if no real confidence exists as to which these are, it is certainly not easy to put confidence in the final estimate of evolutionary relationships. Weighting by constancy within populations, on the other hand, does offer a solution to this problem. The weighting is objective, and it is consistent with evolutionary theory. It is never necessary to ignore characters, but only to assign them some particular weight. The greatest benefits of this weighting method may well be in comprehending the evolution of small groups.

The use of this method places some restrictions on what characters may be used. Only those with a sample variance greater than zero can be weighted in this way. This is not an important consideration in the case of non-meristic (continuously-measured) characters, since the variance of such 
characters will seldom be zero in samples of reasonable size. Meristic characters can attain zero sample variance much more readily. Characters that are indeed invariable within populations are usually those that are useful in studying evolution on a larger scale-and are not particularly useful in studying the relationships between closely related populations. This is true simply because two closely related populations are not expected to differ much in a very conservative character. Such characters can, however, be utilized through an extension of this type of weighting to OTU's of rank higher than population.

Any group of populations at any hierarchical level can be considered as a valid OTU, provided the populations contained in the OTU are more similar to each other than they are to populations in other OTU's. To extend constancy-weighting to OTU's of any rank whatever, we measure the rank of the OTU and the variability between the populations in the OTU of the character that we wish to weight. Differences based on these weighted values can then be used to study relationships between groups of higher rank. Initially, only characters with intrapopulational variances greater than zero can be used. These characters are utilized to group the populations into valid OTU's and to find the rank of the OTU's. To calculate the rank of an OTU, first find the variance between populations for each of the characters used to form the OTU. Each character variance over the OTU is divided by the corresponding intrapopulational character variance, to give "standardized variances." The sum of the standardized variances divided by the number of characters used is a measure of rank of the OTU that takes character conservatism into account. The measure of rank is independent of the original units of measure of the characters, since the units were canceled out of each between-population variance during division by the corresponding within-population variance. Notice that by this mode of calculation a population is always assigned rank 1 , since for every char- acter the variance over the OTU is just the variance within the population. At this point, any character that has a non-zero variance over the OTU can be weighted according to its constancy over the OTU. The square of the weighting coefficient is the rank of the OTU divided by the variance of the given character over the OTU. If all the characters that vary over the OTU are weighted in this way and used to re-calculate the rank of the OTU, the OTU will still have the same rank that it started with. This insures that the right amount of weight is awarded to a given character for a given amount of constancy over an OTU with a particular amount of diversity (rank). The weights arrived at in this manner can be used to study the relationships between separate OTU's.

However, the same weighting cannot be used to study the relationships between populations in the same OTU. To do so would constitute circular reasoning. The greatest weight is assigned to the characters that vary least within OTU's. This is consistent with classical taxonomic practice, differing from it only by greater precision and objectivity. An important point is that weighting by constancy within OTU's of high rank can be used only if there is some assurance that the characters being weighted are non-convergent. A character with widespread convergence within an OTU could receive much too high a weight if it were treated like the other characters. Techniques for detecting convergence must be employed with this weighting procedure, but this matter is too complex to be treated here.

Using this weighting procedure, populations are first grouped on the basis of intrapopulational variability of characters, which is an objectively observable property of those populations. As larger and larger groupings are considered, particular characters are weighted most heavily in those parts of the evolutionary tree where they are most significant, and devaluated or ignored in other parts-but always on the basis of objective calculations. The method 
in effect behaves much as a good taxonomist, selecting the best characters at each level respectively and then reasoning accordingly. Thus, not only does this method assign weights that can be used in inferring evolutionary relationship, but it also assesses the relative importance of different characters at different adaptive stages and the usefulness of characters at different taxonomic levels.

Weighting characters by constancy within populations is an objective technique that is similar in operation to the best statistical procedures. The use of this type of weighting does not introduce subjectivism, circular reasoning, or prior assumptions into the solution of taxonomic problems. The weighting which results is consistent with the population-genetical theory of adaptive and evolutionary mechanisms. Its results are expected to be equivalent to those of weighting by the classical taxonomic concept of conservatism. Constancy of characters within populations can provide evolutionary systematics with an objective means of estimating both conservatism and adaptive importance. The method is expected to be especially useful in small groups and in difficult groups where the application of classical techniques has led to controversy and failed to yield highly reliable results. The technique can be extended to OTU's of rank higher than population, and then it provides a method for assigning weights to characters with zero variance within populations.

\section{ACKNOWLEDGMENTS}

I wish to thank Dr. T. H. Hubbell, Dr. R. R. Miller, and D. L. Hoyt of the University of Michigan Museum of Zoology and particularly Dr. W. H. Wagner of the Department of Botany, The University of Michigan, for criticizing this manuscript. The preparation of this paper was partially supported by grants from the National Science Foundation.

\section{Literature Cited}

Fisher, R. A. 1930. The genetical theory of natural selection (2nd. ed.). Dover, New York, $291 \mathrm{pp}$.

GUTHRIE, R. D. 1965. Variability in characters undergoing rapid evolution, an analysis of Microtus molars. Evolution, 19: 214-233.

MAYR, E. 1963. Animal species and evolution. Harvard University Press, Cambridge, 797 pp.

OLson, E. C. 1964. Morphological integration and the meaning of characters in classification systems. Pp. 115-121 in Phenetic and phylogenetic classification, Systematics Association Publication No. 6, The Systematics Association, London.

Sokal, R. R., and P. H. A. SNeath. 1963. Principles of numerical taxonomy. Freeman, San Francisco, 359 pp. 